Herz 2021 · 46:581-588

https://doi.org/10.1007/s00059-021-05033-2

Eingegangen: 19. Dezember 2020

Überarbeitet: 2. März 2021

Angenommen: 16. März 2021

Online publiziert: 16. April 2021

(c) Der/die Autor(en) 2021

\author{
Harilaos Bogossian ${ }^{1,2}$ (D) - Dimitrios Panteloglou $\cdot$ Zana Karosiene $^{3} \cdot$ \\ Susanne Macher-Heidrich ${ }^{4} \cdot$ Heinz Jürgen Adomeit ${ }^{5} \cdot$ Bernd Lemke $^{3}$. \\ Carsten W. Israel ${ }^{6}$ \\ 'Klinik für Kardiologie und Rhythmologie, Ev. Krankenhaus Hagen, Hagen, Deutschland \\ ${ }^{2}$ Universität Witten/Herdecke, Witten, Deutschland \\ ${ }^{3}$ Klinik für Kardiologie, Elektrophysiologie und Angiologie, Klinikum Lüdenscheid, Lüdenscheid, \\ Deutschland \\ ${ }^{4}$ Ärztekammer Nordrhein, Düsseldorf, Deutschland \\ ${ }^{5}$ Ärztekammer Westfalen-Lippe, Münster, Deutschland \\ ${ }^{6}$ Klinik für Kardiologie, Evangelisches Klinikum Bethel, Bielefeld, Deutschland
}

\title{
Peripoperative Mortalität nach ICD-Implantation
}

In der vorliegenden Arbeit sollen aus den Datensätzen der QS NRW die Baseline- und Implantationsparameter identifiziert werden, die mit einer erhöhten perioperativen Mortalität nach ICD-Implantation assoziiert sind.

\section{Methode}

In der aktuellen Studie wurden die Datensätze der ICD-Implantationen in NRW von 2010 bis 2012 ausgewertet. Für die Auswertung wurde das Datenmaterial verwendet, das der Geschäftsstelle der QS NRW im Rahmen der gesetzlichen stationären externen Qualitätssicherung vorliegt (insgesamt 18.625 Implantationen: 8507 Einkammer-ICD, 4364 Zweikammer-ICD, 5596 kardiale Resynchronisationstherapie [CRT], 158 sonstige ICD). Aus dem Datenmaterial wurden folgende Parameter über den stationären Aufenthalt der Patienten analysiert:

- Patientencharakteristika: Alter, Geschlecht, NYHA(New York Heart Association)-Klasse, ASA(American Society of Anesthesiologists)Klassifikation [5], Diabetes mellitus (vorhanden/nicht vorhanden/ insulinpflichtig), Niereninsuffizienz (Kreatinin: $<1,5 / 1,5-2,5 />2,5 \mathrm{mg} / \mathrm{dl}$ ohne und mit Dialyse), Vorliegen einer koronaren Herzkrankheit (KHK) oder Kardiomyopathie;

- Echokardiographie (linksventrikuläre Ejektionsfraktion, LVEF);
- Indikation zur ICD-Implantation;

- perioperative Komplikationen während des stationären Aufenthalts (Reanimation, Pneumothorax, Hämatothorax, Perikarderguss, Taschenhämatom, Sondendislokation, Wundinfektion, sonstige);

- Art der Entlassung (nach Hause, Verlegung in anderes Krankenhaus, Rehabilitation oder Heim, Patient verstorben).

Im „strukturierten Dialog“, werden im Rahmen des Indikators „Mortalität“ verbindlich Fragebögen ausgefüllt, die sich auf verstorbene Patienten beziehen.

Mit Einschätzung des Operateurs findet eine Beurteilung darüber statt, ob aufgetretene Komplikationen ursächlich oder mitursächlich für den Tod des Patienten verantwortlich waren. Die Komplikationsart und der zeitliche Zusammenhang zur Implantation müssen angegeben werden. Sollte weder eine Komplikation noch eine Herzrhythmusstörung für den Tod des Patienten verantwortlich sein, muss dies ausdrücklich vermerkt werden. Ein unsicherer Zusammenhang zwischen dem Tod des Patienten und einer Komplikation der ICDImplantation wird ebenfalls dokumentiert. Zudem macht der Operateur Angaben zu Begleiterkrankungen, die im Rahmen der ICD-Implantation hinsichtlich der Prognose sowie des peri- und postoperativen Verlaufs einen beeinflus- 

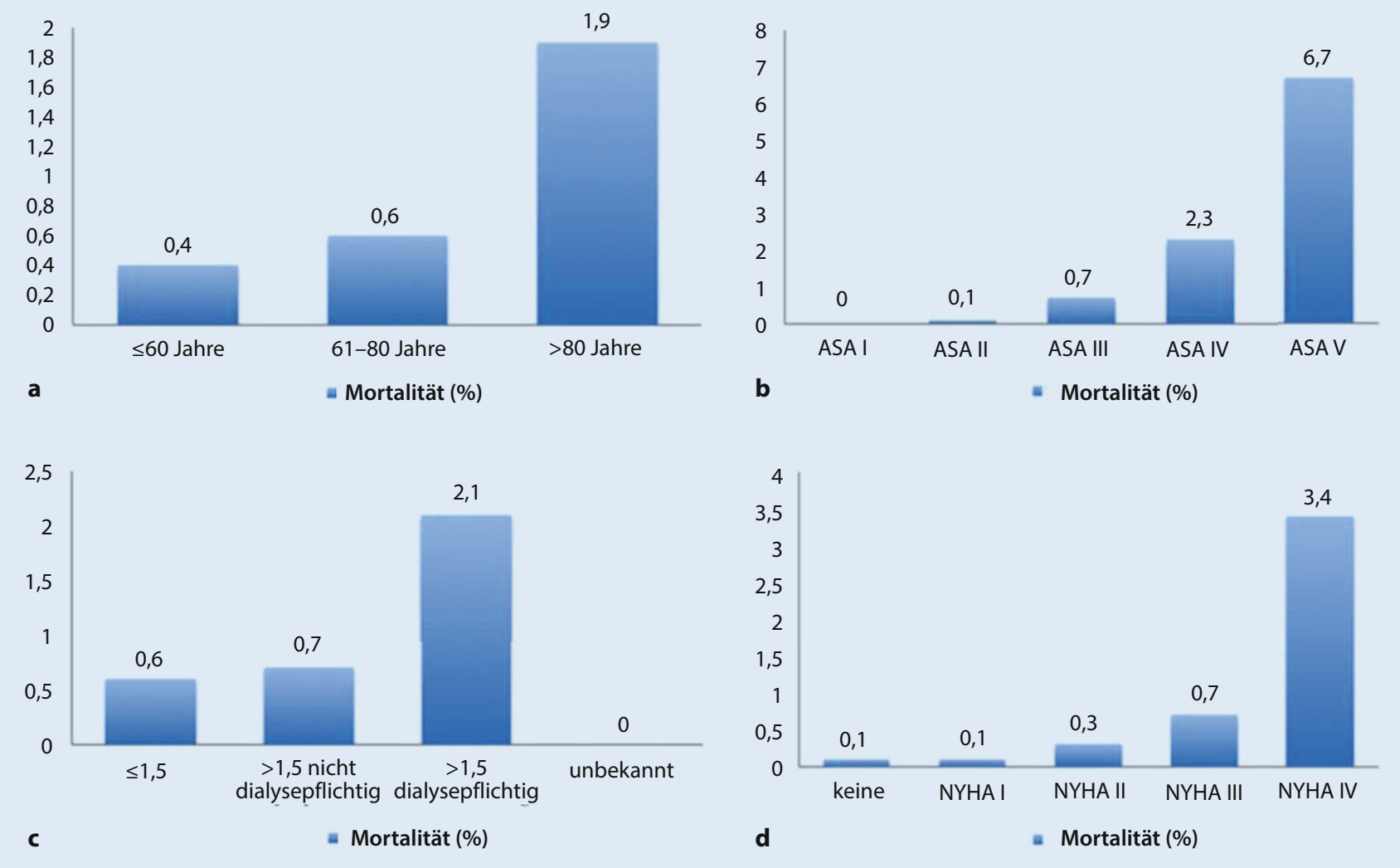

Abb. 1 ॥ Mortalität in Abhängigkeit von Alter (a), ASA(American Society of Anesthesiologists)-Klasse (b), Niereninsuffizienz (c) und NYHA(New York Heart Association)-Stadium (d; Ordinate: Mortalität in \%, Abszisse: unterschiedliche Gruppen [siehe Text])

senden Charakter besitzen. Die Angaben erheben keinen Anspruch auf Vollständigkeit. Der Operateur muss dokumentieren, ob nach der ICD-Implantation ein Röntgenthorax, eine Echokardiographie und eine ICD-Kontrolle stattgefunden haben, und dabei den zeitlichen Zusammenhang mit der Implantation berücksichtigen. Für die Analyse des „strukturierten Dialogs" liegen nur die Daten für die Jahre von 2010 bis 2011 vor.

\section{Statistische Analyse}

Es erfolgte eine deskriptive Analyse des Datenmaterials. Die statistische Signifikanz der Ergebnisse wurde mittels ChiQuadrat-Tests nach Pearson berechnet (Signifikanzniveau: $p \leq 0,05$ ). Die statistische Auswertung der Ergebnisse erfolgte unter Verwendung der Statistik- und Analysesoftware SPSS. Die Darstellung erfolgt in tabellarischer Form sowie in Form von Balkendiagrammen.

\section{Ergebnisse}

\section{Patientenkollektiv und Altersverteilung}

In den Jahren von 2010 bis 2012 erhielten 18.625 Patienten einen ICD. Während des stationären Aufenthalts verstarben 118 Patienten $(0,6 \%)$. Beim weiblichen Geschlecht zeigt sich eine signifikant höhere Mortalität (männlich: 79 von 14.508 [0,54\%]; weiblich: 39 von 4117 [0,95\%; $p=0,004])$.

Das Alter der Patienten betrug zum Zeitpunkt der ICD-Implantation im Mittel 66 Jahre (Median: 69; Minimum: $<1$, Maximum: 91 Jahre), das der Verstorbenen im Mittel 71 Jahre (Median: 74; Minimum: 25, Maximum: 88 Jahre). Die Patienten wurden in die folgenden Altersgruppen eingeteilt:

- $\leq 60$ Jahre $(n=5212 ; 27,98 \%)$,

- 61-80 Jahre ( $n=12.020 ; 64,54 \%)$,

- $>80$ Jahre $(n=1393 ; 7,48 \%)$.
Die Überlebenswahrscheinlichkeit zwischen den ersten beiden Altersgruppen zeigte keinen signifikanten Unterschied $(p=0,0748)$. Die Patienten der dritten Altersgruppe hatten gegenüber den beiden jüngeren Gruppen eine signifikant höhere Mortalität (jeweils $p<0,001$; 0 Abb. 1 ; - Tab. 1).

\section{ASA-Klassifikation, NYHA-}

Stadium und linksventrikuläre Ejektionsfraktion

Die Mortalität war bei Patienten mit höherer ASA-Klasse gegenüber Patienten mit niedriger ASA-Klasse signifikant höher. Wegen geringer Fallzahl in den ASAKlassen I und V konnte keine adäquate Signifikanzberechnung erfolgen. Die Ermittlung der Signifikanzen zwischen den Gruppen ASA II-IV ergab folgende Werte (• Abb. 1; • Tab. 1):

- ASA II zu III: $p<0,001$;

- ASA II zu IV: $p<0,001$;

- ASA III zu IV: $p<0,001$. 
Herz 2021 • 46:581-588 https://doi.org/10.1007/s00059-021-05033-2

(c) Der/die Autor(en) 2021

\section{H. Bogossian · D. Panteloglou · Z. Karosiene · S. Macher-Heidrich · H. J. Adomeit · B. Lemke · C. W. Israel}

\section{Peripoperative Mortalität nach ICD-Implantation}

\section{Zusammenfassung}

Hintergrund. Implantierbare KardioverterDefibrillatoren (ICD) sind zum Schutz vor plötzlichem Herztod bei Patienten mit primäroder sekundärprophylaktischer Indikation etabliert. Wie bei allen komplexen operativen Verfahren verbleibt auch bei der ICDImplantation ein Risiko für Komplikationen bis hin zum Tod. Gegenstand der vorliegenden Arbeit ist es, anhand der Datensätze zur obligaten Qualitätssicherung in NordrheinWestfalen die prozedurbezogene Mortalität nach ICD-Implantation zu analysieren. Methoden. Aus den Datensätzen erfolgte eine Analyse der stationären Todesfälle bei allen 18.625 ICD-Implantationen der Jahre 2010 bis 2012.
Ergebnisse. Während des stationären Aufenthalts verstarben 118 Patienten $(0,6 \%)$ nach ICD-Implantation. Patienten im Alter über 80 Jahre (7\%) zeigten eine höhere Mortalität $(1,9 \%$ vs. $0,5 \%$ bei $<80$-jährigen Patienten, $p>0,001)$, ebenso Frauen $(0,95 \%$ vs. $0,54 \%$ bei Männern; $p=0,004)$ und Patienten mit hoher NYHA(New York Heart Association)-Klasse (0,3\% bei NYHA II, $0,7 \%$ bei NYHA III, 3,4\% bei NYHA IV; $p<0,001$ für alle Vergleiche). Das Vorliegen von Diabetes mellitus (23\% des Kollektivs) beeinflusste die perioperative Letalität nicht, während eine dialysepflichtige Niereninsuffizienz eine signifikant erhöhte Mortalität aufwies $(p<0,001$ gegenüber Patienten mit Kreatinin $\leq 1,5 \mathrm{mg} / \mathrm{dl} ; p=0,002$ gegenüber nicht dialysepflichtigen Patienten mit Kreatinin $>1,5 \mathrm{mg} / \mathrm{dl})$. Patienten mit sekundärprophylaktischer ICD-Indikation wiesen eine signifikant höhere Mortalität auf $(1,2 \%$ vs. $0,4 \% ; p<0,001)$, die sich beim Auftreten von Komplikationen von $0,6 \%$ auf $3,7 \%$ erhöhte $(p<0,001)$.

Schlussfolgerung. Die operationsbezogene Mortalität bei ICD-Implantation ist bei Patienten über 80 Jahre, hoher NYHAKlasse, Dialysepflicht, sekundärprophylaktischer Indikation und nach Auftreten von Komplikationen erhöht.

Schlüsselwörter Defibrillatoren · Operation · Komplikationen Risikofaktoren · Qualitätssicherung

\section{Perioprocedural mortality after ICD implantation}

\section{Abstract}

Background. Implantable cardioverter defibrillators (ICD) represent an established treatment in preventing sudden cardiac death in patients with indications for primary or secondary prophylaxis. As for all complex surgical procedures there remains a risk for the occurrence of complications including death also for ICD implantation. The aim of the present study was to analyze the procedure-related mortality in patients after ICD implantation using the data from the obligatory quality assurance program in North-Rhine/Westphalia.

Methods. Data of all 18,625 patients from the quality assurance datasets who underwent ICD implantation in the years 2010-2012 were analyzed.
Results. During the in-hospital stay 118 patients $(0.6 \%)$ died after ICD implantation. Patients $>80$ years old had a higher mortality ( $1.9 \%$ vs. $0.5 \%$ in patients $<80$ years old, $p<0.001)$ as well as women $(0.95 \%$ vs. $0.54 \%$ in men, $p=0.004$ ) and patients with higher New York Heart Association (NYHA) class (0.3\% for NYHA II, $0.7 \%$ for NYHA III, 3.4\% for NYHA IV, $p<0.001$ for all comparisons). The presence of diabetes mellitus ( $23 \%$ of the collective) did not influence the perioperative mortality, whereas renal failure requiring dialysis showed a significantly increased mortality $(p<0.001$ compared to patients with creatinine $\leq 1.5 \mathrm{mg} / \mathrm{dl}$ and $p=0.002$ for patients with creatinine $>1.5 \mathrm{mg} / \mathrm{dl}$ not requiring dialysis). Patients with indications for
ICD secondary prophylaxis had a significantly higher mortality $(1.2 \%$ vs. $0.4 \%, p<0.001)$, which increased from $0.6 \%$ to $3.7 \%(p<0.001)$ with the occurrence of complications.

Conclusion. The procedure-related mortality after ICD implantation is increased in patients over 80 years of age, higher NYHA class, patients requiring dialysis, in secondary prevention indications and after the occurrence of perioperative complications.

Keywords

Defibrillators · Surgery · Complications · Risk factors · Quality assurance
Die Mortalität bei Patienten höherer NYHA-Klassen war signifikant höher als bei Patienten niedriger NYHA-Klassen. Bei NYHA I erfolgte wegen der geringeren Fallzahl keine Signifikanzberechnung. Die übrigen Signifikanzen wurden wie folgt berechnet (• Abb. 1; - Tab. 1):

- NYHA II und NYHA III: $p<0,001$;

- NYHA II und NYHA IV: $p<0,001$;

- NYHA III und NYHA IV: $p<0,001$.
Bei der Unterteilung der Patienten nach ihrer EF wurden 4 Gruppen gebildet (EF $>50 \%$, EF $>35-50$, EF $\leq 35$, EF unbekannt; - Tab. 1). Entsprechend der ICDIndikationen war die Gruppe von Patienten mit einer EF von $35 \%$ oder weniger am größten (> $80 \%$ der Patienten). Die Mortalität zeigte keine signifikanten Unterschiede zwischen den hier gebildeten Gruppen (• Tab. 1):

- $\mathrm{EF} \leq 35 \%$ vs. $>35-50 \%: p=0,6506$;

- $\mathrm{EF} \leq 35 \%$ vs. $>50 \%: p=0,4$.
Komorbidität, Kardiomyopathien, führende Indikation und Komplikationen

Die Analyse der perioperativen Mortalität der Patienten mit und ohne Diabetes mellitus zeigte keine signifikanten Unterschiede (• Tab. 1):

- kein Diabetes mellitus vs. nicht insulinpflichtiger Diabetes mellitus: $p=0,452$; 


\begin{tabular}{|c|c|c|c|c|}
\hline & Gesamt & Gesamt \% & Tote & Tote \% \\
\hline \multicolumn{5}{|l|}{ Alter } \\
\hline$\leq 60$ Jahre & 5212 & 27,98 & 20 & 0,4 \\
\hline 61-80 Jahre & 12.020 & 64,54 & 72 & 0,6 \\
\hline$>80$ Jahre & 1393 & 7,48 & 26 & 1,9 \\
\hline \multicolumn{5}{|c|}{$\begin{array}{l}\leq 60 \text { Jahre und }>80 \text { Jahre: } p<0,001 \\
61-80 \text { Jahre und }>80 \text { Jahre: } p<0,001 \\
\leq 60 \text { Jahre und } 61-80 \text { Jahre: } p=0,0748\end{array}$} \\
\hline \multicolumn{5}{|l|}{ Geschlecht } \\
\hline Männlich & 14.508 & 77,9 & 79 & 0,5 \\
\hline Weiblich & 4117 & 22,1 & 39 & 1 \\
\hline \multicolumn{5}{|c|}{ Männlich vs. Weiblich: $p=0,004$} \\
\hline \multicolumn{5}{|c|}{ ASA-Klassifikation } \\
\hline ASA I & 429 & 2,3 & 0 & 0 \\
\hline ASA II & 4817 & 25,9 & 6 & 0,1 \\
\hline ASA III & 12.268 & 65,9 & 86 & 0,7 \\
\hline ASA IV & 1096 & 5,9 & 25 & 2,3 \\
\hline ASA V & 15 & 0,1 & 1 & 6,7 \\
\hline \multicolumn{5}{|c|}{$\begin{array}{l}\text { Wegen geringer Fallzahl wird für die ASA-Klassen I und V keine Signifikanz angegeben. } \\
\text { ASA II vs. III: } p<0,001 \\
\text { ASA II vs. IV: } p<0,001 \\
\text { ASA III vs. IV: } p<0,001\end{array}$} \\
\hline \multicolumn{5}{|l|}{ NYHA-Klasse } \\
\hline Keine & 990 & 5,3 & 1 & 0,1 \\
\hline NYHA I & 1148 & 6,2 & 1 & 0,1 \\
\hline NYHA II & 6229 & 33,4 & 21 & 0,3 \\
\hline NYHA III & 9517 & 51,1 & 70 & 0,7 \\
\hline NYHA IV & 741 & 4 & 25 & 3,4 \\
\hline \multicolumn{5}{|c|}{$\begin{array}{l}\text { NYHA II vs. NYHA III: } p<0,001 \\
\text { NYHA II vs. NYHA IV: } p<0,001 \\
\text { NYHA III vs. NYHA IV: } p<0,001\end{array}$} \\
\hline \multicolumn{5}{|c|}{ Ejektionsfraktion (EF) } \\
\hline EF unbekannt & 343 & 1,8 & 5 & 1,5 \\
\hline$E F>50 \%$ & 1328 & 7,1 & 6 & 0,5 \\
\hline EF 50 bis $>35 \%$ & 1981 & 10,6 & 11 & 0,6 \\
\hline$E F \leq 35 \%$ & 14.973 & 80,4 & 96 & 0,6 \\
\hline \multicolumn{5}{|c|}{$\begin{array}{l}\mathrm{EF} \leq 35 \% \text { und } 35-50 \%: p=0,6506 \\
\mathrm{EF} \leq 35 \% \text { und }>50 \%: p=0,4\end{array}$} \\
\hline
\end{tabular}

- kein Diabetes mellitus vs. insulinpflichtiger Diabetes mellitus: $p=0,892$;

- kein Diabetes mellitus vs. Diabetes mellitus (insulinpflichtig und nicht insulinpflichtig): $p=0,61$.

Dagegen war die dialysepflichtige Niereninsuffizienz sowohl gegenüber Patienten ohne Niereninsuffizienz als auch gegenüber nicht dialysepflichtigen niereninsuffizienten Patienten mit einer tienten mit und ohne KHK war gleich

(• Tab. 2):

- KHK mit Myokardinfarkt in der Anamnese vs. Patienten ohne KHK: $p=0,581$;

- KHK mit Myokardinfarkt in der Anamnese vs. KHK ohne Myokardinfarkt in der Anamnese: $p=0,375$.

Hinsichtlich der Implantationsindikation zeigte sich die Mortalität bei Patienten mit primärpräventiver ICD-Indikation gegenüber der sekundärpräventiven Indikation niedriger ( $p \leq 0,001$; • Abb. 2a).

Bei 1,9\% der Patienten traten perioder postoperative Komplikationen (kardiopulmonale Reanimation, Pneumothorax, Hämatothorax, Perikarderguss, Taschenhämatom, Sondendislokation, Wundinfektion oder sonstige) auf; bei diesen Patienten lag die Mortalität mit 3,7\% signifikant höher als bei Patienten ohne perioperative Komplikationen (0,6\%; $p<0,001 ; \bullet$ Abb. 2b).

\section{Prozedurbedingte Mortalität}

Für die Jahre 2010 und 2011 liegen die Fragebögen des "strukturierten Dialogs“ vor. Demnach wurden die Fälle der 89 verstorbenen Patienten (von insgesamt 12.121 ICD-versorgten Patienten in den Jahren 2010-2011) im Einzelnen mit den implantierenden Kliniken/ Operateuren diskutiert. Daraus resultierte, dass nur 4 Patienten $(0,033 \%)$ unmittelbar perioperativ verstorben sind.

\section{Diskussion}

Die vorliegende Analyse von fast 20.000 ICD-Implantationen zeigt, dass eine ICD-Implantation bei folgenden Patienten bzw. Bedingungen mit einer erhöhten Mortalität noch während des stationären Aufenthalts nach Implantation assoziiert ist:

- Alter > 80 Jahre,

- weibliches Geschlecht,

- schlechterer Allgemeinzustand bzw. fortgeschrittene Herzinsuffizienzsymptomatik (ASA-Klasse, NYHAKlasse),

- terminale Niereninsuffizienz mit Notwendigkeit einer Dialyse, 


\begin{tabular}{|c|c|c|c|c|}
\hline & Gesamt & Gesamt \% & Tote & Tote \% \\
\hline \multicolumn{5}{|c|}{ Diabetes mellitus (DM) } \\
\hline Kein DM & 14.259 & 76,6 & 88 & 0,6 \\
\hline $\begin{array}{l}\text { Nicht insulin- } \\
\text { pflichtiger DM }\end{array}$ & 2837 & 15,2 & 21 & 0,7 \\
\hline $\begin{array}{l}\text { Insulinpflichtiger } \\
\text { DM }\end{array}$ & 1529 & 8,2 & 9 & 0,6 \\
\hline \multicolumn{5}{|c|}{$\begin{array}{l}\text { Kein Diabetes mellitus vs. Diabetes mellitus (nicht insulinpflichtig): } p=0,452 \\
\text { Kein Diabetes mellitus vs. Diabetes mellitus (insulinpflichtig): } p=0,892 \\
\text { Kein Diabetes mellitus vs. Diabetes mellitus (insulinpflichtig und nicht insulinpflichtig): } p=0,61\end{array}$} \\
\hline \multicolumn{5}{|l|}{ Niereninsuffizienz } \\
\hline $\begin{array}{l}\text { Kreatinin } \\
\leq 1,5 \mathrm{mg} / \mathrm{dl}\end{array}$ & 14.856 & 79,8 & 88 & 0,6 \\
\hline $\begin{array}{l}\text { Kreatinin } \\
>1,5 \mathrm{mg} / \mathrm{dl} \text { (nicht } \\
\text { dialysepflichtig) }\end{array}$ & 3177 & 17,1 & 21 & 0,7 \\
\hline $\begin{array}{l}\text { Kreatinin } \\
>1,5 \mathrm{mg} / \mathrm{dl} \text { (dialy- } \\
\text { sepflichtig) }\end{array}$ & 423 & 2,3 & 9 & 2,1 \\
\hline Unbekannt & 169 & 0,9 & 0 & 0 \\
\hline \multicolumn{5}{|c|}{$\begin{array}{l}\text { Kreatinin } \leq 1,5 \mathrm{mg} / \mathrm{dl} \text { vs. }>1,5 \mathrm{mg} / \mathrm{dl} \text { (dialysepflichtig): } p<0,001 \\
\text { Kreatinin } \leq 1,5 \mathrm{mg} / \mathrm{dl} \mathrm{vs.}>1,5 \mathrm{mg} / \mathrm{dl} \text { (nicht dialysepflichtig): } p=0,65 \\
\text { Kreatinin }>1,5 \mathrm{mg} / \mathrm{dl} \text { (nicht dialysepflichtig) vs. }>1,5 \mathrm{mg} / \mathrm{dl} \text { (dialysepflichtig): } p=0,002\end{array}$} \\
\hline \multicolumn{5}{|c|}{ Koronare Herzkrankheit (KHK) } \\
\hline $\begin{array}{l}\text { KHK ohne Ml in } \\
\text { der Anamnese }\end{array}$ & 4473 & 24 & 25 & 0,6 \\
\hline $\begin{array}{l}\text { KHK mit MI in der } \\
\text { Anamnese }\end{array}$ & 7357 & 39,5 & 51 & 0,7 \\
\hline Nein & 6795 & 36,5 & 42 & 0,6 \\
\hline \multicolumn{5}{|c|}{$\begin{array}{l}\text { KHK mit MI vs. keine KHK: } p=0,581 \\
\text { KHK mit MI vs. KHK ohne MI: } p=0,375\end{array}$} \\
\hline MI Myokardinfarkt & & & & \\
\hline
\end{tabular}

Tab. 3 Verteilung der kardialen Grunderkrankung und Einfluss auf die Mortalität

\begin{tabular}{|lll|l|l}
\hline Kardiale Grunderkrankung & Gesamt & Gesamt \% & Tote & Tote \% \\
\hline Keine & 481 & 2,6 & 0 & 0 \\
\hline ICM & 10.506 & 56,4 & 73 & 0,7 \\
DCM & 5939 & 31,9 & 32 & 0,5 \\
\hline Hypertensive Herzkrankheit & 329 & 1,8 & 1 & 0,3 \\
Erworbene Herzklappenfehler & 172 & 0,9 & 3 & 1,7 \\
\hline Angeborene Herzklappenfehler & 60 & 0,3 & 0 & 0 \\
Brugada-Syndrom & 91 & 0,5 & 0 & 0 \\
Kurzes QT-Syndrom & 0 & 0 & 0 & 0 \\
\hline Langes QT-Syndrom & 144 & 0,8 & 0 & 0 \\
\hline HCM & 397 & 2,1 & 2 & 0,5 \\
ARVC & 48 & 0,3 & 0 & 0 \\
Sonstige & 458 & 2,5 & 7 & 1,5 \\
\hline ICM vs. DCM: $p=0,228$ & \multicolumn{5}{|l}{} \\
\hline ICM ischämische Kardiomyopathie, DCM dilatative Kardiomyopathie, HCM hypertrophe Kardiomyopa- \\
thie, ARVC arrhythmogene rechtsventrikuläre Kardiomyopathie
\end{tabular}

- sekundärprophylaktische ICD-Indikation (•Tab. 4),

- Auftreten von Komplikationen während oder nach der Implantation (• Tab. 4).

Die Beurteilung von QS-Daten wird kontrovers diskutiert [6]. Die vorgestellten Daten sind jedoch aufgrund ihrer praktisch lückenlosen Abbildung der Mortalität bei einer so großen Zahl konsekutiver, nichtselektionierter ICD-Implantationen von beträchtlicher klinischer Relevanz. Nicht zuletzt weil „Tod“ einen „harten“"Endpunkt darstellt, der aufgrund der Verknüpfung der Krankenhaussoftware überprüfbar ist und kaum fehldokumentiert werden kann, ist die Validität der Daten als sehr hoch einzustufen.

Die Ergebnisse können für operierende Ärzte von hoher Relevanz sein, da sie helfen, Patienten zu identifizieren, die ein besonderes Risiko haben, noch während des stationären Aufenthalts zu versterben und von der ICD-Implantation keinen Nutzen zu erlangen. Die Ergebnisse können insbesondere für die Patientenaufklärung hilfreich sein, das individuelle perioperative Risiko genauer einzustufen.

Die Indikationen zur ICD-Implantation sind sowohl zur Primär- als auch zur Sekundärprävention etabliert [2, 7-10], obwohl sie auf älteren Studien basieren. Es liegen viele Langzeitdaten zu Komplikationen und Mortalität vor [11-13]. Hinsichtlich der perioperativen Mortalität basieren die Daten insbesondere auf Registerdaten $[4,14,15]$. Die vorliegende Auswertung der Datensätze der QS NRW ist vergleichbar mit ausländischen Ergebnissen, denen zufolge die Mortalität bei der ICD-Implantation tatsächlich niedriger liegt als bei Schrittmacherimplantationen [3]. Über die Ursachen dessen kann nur spekuliert werden (jüngeres Alter der Patienten mit ICD- im Vergleich zur Schrittmacherimplantation, Operation in erfahreneren Institutionen bzw. von erfahreneren Implanteuren, geringere Komorbidität?). Im Unterschied zur Schrittmacherimplantation fordert die aktuelle QS für Patienten mit ICD-Implantation eine mindestens 1-jährige Lebenserwartung in gutem funktionellen Status. 


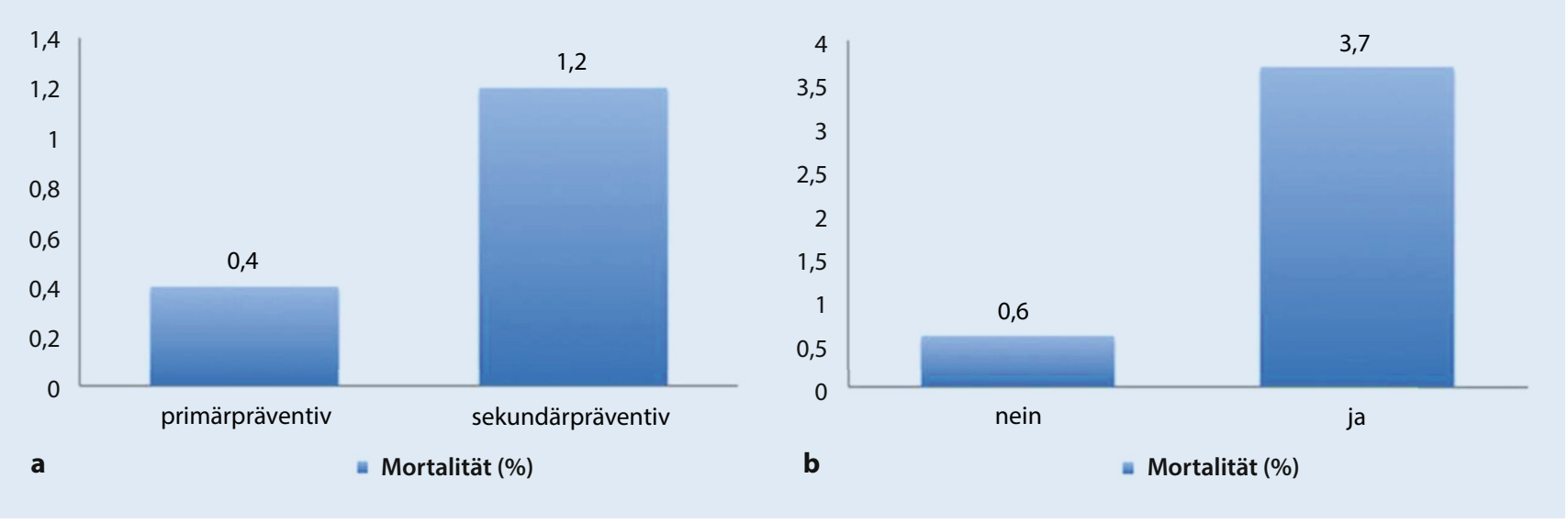

Abb. 2 ム Mortalität in \%, bezogen auf die Indikation (a) und in Abhängigkeit vom Vorhandensein perioperativer Komplikationen (b)

Das geschlechtsspezifische Outcome variiert in der Literatur stark. Neben Studien, in denen kaum relevante geschlechtsspezifische Unterschiede beobachtet wurden [16], konnte in anderen gezeigt werden, dass Komplikationen bei Frauen häufiger auftreten [17]. Hierbei sind insbesondere der Pneumothorax und die Perikardtamponade zu nennen [18]. In der vorliegenden Auswertung zeigte sich eine perioperativ signifikant erhöhte Mortalität bei Frauen. Kanadische Kardiologen haben die ICDVersorgung von Männern und Frauen analysiert und berichtet, dass - trotz Adjustierung - Männer nach Myokardinfarkt oder mit Herzinsuffizienz 3-mal häufiger mit einem ICD versorgt werden [19]. Dies lässt vermuten, dass Frauen nur bei schwerer Herzinsuffizienz versorgt werden und somit eine höhere Anfälligkeit haben könnten. Eine höhere Rate an Komplikationen bei Frauen (Blutungen, Wundheilungsstörungen etc.) wurde in vielen Studien mit invasiven bzw. operativen Therapien nachgewiesen.

Al-Khatib et al. haben wesentliche Risikofaktoren der Sterblichkeit definiert. Hierunter zählen unter anderem [17]:

- Herzinfarkt in der Anamnese,

- Diabetes mellitus,

- Niereninsuffizienz und Herzinsuffizienz.

Hinsichtlich der perioperativen Mortalität haben sich in den aktuell ausgewerteten Daten nur die dialysepflichtige Niereninsuffizienz und die Herzinsuffi- zienz, basierend auf der NYHA-Klasse, aber nicht die EF als signifikante Marker bestätigt. Trotzdem sollte Dialysepatienten eine ICD-Implantation nicht vorenthalten werden, da auch neue Daten zeigen, dass Dialysepatienten von einer sekundärprophylaktischen ICD-Implantation profitieren [20].

Die vorliegenden Daten zeigen ein 3-fach erhöhtes Risiko bei der sekundärprophylaktischen ICD-Implantation. Dies ist am ehesten auf dem Boden der akuten Arrhythmielast mit möglicherweise auch reduziertem Status der Patienten vor der Operation (z. B. nach Reanimation) zu erklären. Daher sollte der Zeitpunkt der ICD-Implantation bei diesen Patienten kritisch gewählt werden. Das Hauptargument gegen ein längeres Zuwarten ist die Gefahr eines Arrhythmierezidivs. Allerdings sollte in einem stabilen Zustand und nicht unmittelbar nach einem VT(ventrikuläre Tachykardie)-Sturm operiert werden. Dabei gilt es abzuwägen, ob im stationären Setting eine protrahierte Implantation erfolgen oder sogar nach Versorgung mit tragbarer Defibrillatorweste der Zeitpunkt der Operation weiter verschoben werden sollte. Auch eine frühzeitige VT-Ablation vor der ICD-Implantation ist Gegenstand aktueller Studien [21].

Unsere Daten sollten dazu genutzt werden, bei multimorbiden Patienten bereits im Vorfeld an ein erhöhtes Risiko für Komplikationen und perioperative Mortalität zu denken und daher bei jedem Schritt der Implantation beson- dere Sorgfalt walten $\mathrm{zu}$ lassen. Dies bekräftigen auch die Daten aus dem „strukturierten Dialog“. Einerseits ist das sehr niedrige operationsassoziierte Risiko als positiv zu bewerten, und es bestätigt die sichere Durchführung der Implantationen, andererseits rückt der Appell für die korrekte Auswahl der Patienten weiter in den Vordergrund, da die Mortalität überwiegend durch die Komorbidität der Patienten während des stationären Aufenthalts erklärt ist.

Bei der Implantation von Ein- und Zweikammer-ICD ist eine Komplikationsrate von 1,0-4,5\% beschrieben [4]. In der aktuellen Auswertung bei $18.625 \mathrm{~Pa}$ tienten betrug die Inzidenz von Komplikationen 1,9\%. Diese Patienten bedürfen postoperativ einer besonderen Aufmerksamkeit, da ein signifikant (rechnerisch 6-fach) erhöhtes Risiko einer perioperativen Mortalität besteht (3,7\% vs. $0,6 \%$; $p<0,001)$.

Das perioperative Mortalitätsrisiko ist in der vorliegenden Analyse bei Patienten mit sekundärprophylaktischer Indikation mit 1,2\% signifikant höher als bei solchen mit primärprophylaktischer Indikation $(0,4 \% ; p \leq 0,001)$. Dies beschreibt allerdings nur die akute Beobachtung. Hinsichtlich des Langzeitbeobachtung liegen in der Literatur auch entgegengesetzte Daten vor. So besteht bei Patienten nach primärprophylaktischer Implantation (nach der ersten ICD-Auslösung) eine höhere Arrhythmielast als bei Patienten nach sekundärprophylaktischer Implantation [22]. 
Tab. 4 Mortalität in Abhängigkeit von der ICD(implantierbarer Kardioverter-Defibrillator)-Indikation und vom Auftreten von Komplikationen

\begin{tabular}{|c|c|c|c|c|}
\hline Führende Indikation & Gesamt & Gesamt \% & Tote & Tote \% \\
\hline Primärpräventiv & 13.255 & 71,2 & 55 & 0,4 \\
\hline Sekundärpräventiv & 5370 & 28,8 & 63 & 1,2 \\
\hline \multicolumn{5}{|c|}{ Primärpräventiv vs. sekundärpräventiv: $p \leq 0,001$} \\
\hline \multicolumn{5}{|l|}{ Komplikationen } \\
\hline Nein & 18.271 & 98,1 & 105 & 0,6 \\
\hline Ja & 354 & 1,9 & 13 & 3,7 \\
\hline
\end{tabular}

Auftreten von Komplikationen vs. keine Komplikationen: $p<0,001$

Zusammengefasst präsentieren die analysierten Daten einer großen Patientenkohorte, dass es, obwohl die perioperative Mortalität bei der ICD-Implantation mit $0,6 \%$ niedrig ist, Patienten mit deutlich erhöhtem Risiko gibt, die identifiziert und entsprechend protektiv behandelt werden sollten. Aufgrund von sowohl neuen kritischen Daten bezüglich der ICD-Therapie [23, 24], verbesserter medikamentöser Herzinsuffizienztherapie, verbesserter Interventions- und Ablationstherapie als auch der Option, längere zeitliche „Risikofenster“ mit tragbaren Defibrillatoren zu überbrücken, sollten insbesondere bei Risikopatienten alle Möglichkeiten genutzt werden, um eine ICD-Implantation, sofern dies sinnvoll sein kann, zu vermeiden.

\section{Limitationen}

Die Ergebnisse liefern wichtige Erkenntnisse über postoperative Komplikationen und das Risikomanagement von ICD-Patienten, die jedoch auf die durch die QS erfassten Daten limitiert sind. Die Analyse ist auf die Krankenhausmortalität beschränkt, und es kann keine Aussage über die Mortalität nach Entlassung oder im Follow-up getroffen werden. Dafür werden hier jedoch sicher alle Todesfälle zum Entlassungszeitpunkt erfasst. Natürlich können nach Entlassung und darüber hinaus noch Patienten verstorben sein, aber deren Anzahl ist als gering einzuschätzen. Diese Limitation resultiert aus der gewählten Methodik, die durch die QS vorgegeben ist.

\section{Fazit für die Praxis}

\section{- Hinsichtlich der perioperativen Mortalität besteht ein signifi-}

kant erhöhtes Risiko an einer ICD(implantierbarer KardioverterDefibrillator)-Operation zu versterben, wenn die Patienten älter als 80 Jahre oder weiblich sind, ein fortgeschrittenes NYHA(New York Heart Association)-Stadium oder eine dialysepflichtige Niereninsuffizienz aufweisen.

- Patienten, die einen ICD aufgrund einer sekundärprophylaktischen Indikation erhalten, haben ein höheres perioperatives Mortalitätsrisiko.

- Wie bei der Herzschrittmacherimplantation ist auch bei der ICDImplantation das Auftreten von Komplikationen ein Risikomarker für eine höhere perioperative Mortalität.

\section{Korrespondenzadresse}

PD Dr. Harilaos Bogossian

Klinik für Kardiologie und Rhythmologie, Ev. Krankenhaus Hagen

Brusebrinkstr. 20, 58135 Hagen, Deutschland bogossianh@esv.de

Funding. Open Access funding enabled and organized by Projekt DEAL.

\section{Einhaltung ethischer Richtlinien}

Interessenkonflikt. H. Bogossian, D. Panteloglou, Z. Karosiene, S. Macher-Heidrich, H.J. Adomeit, B. Lemke und C.W. Israel geben an, dass kein Interessenkonflikt besteht.

Für diesen Beitrag wurden von den Autoren keine Studien an Menschen oder Tieren durchgeführt. Für die aufgeführten Studien gelten die jeweils dort angegebenen ethischen Richtlinien.

Open Access. Dieser Artikel wird unter der Creative Commons Namensnennung 4.0 International Lizenz veröffentlicht, welche die Nutzung, Vervielfältigung, Bearbeitung, Verbreitung und Wiedergabe in jeglichem Medium und Format erlaubt, sofern Sie den/die ursprünglichen Autor(en) und die Quelle ordnungsgemäß nennen, einen Link zur Creative Commons Lizenz beifügen und angeben, ob Änderungen vorgenommen wurden.

Die in diesem Artikel enthaltenen Bilder und sonstiges Drittmaterial unterliegen ebenfalls der genannten Creative Commons Lizenz, sofern sich aus der Abbildungslegende nichts anderes ergibt. Sofern das betreffende Material nicht unter der genannten Creative Commons Lizenz steht und die betreffende Handlung nicht nach gesetzlichen Vorschriften erlaubt ist, ist für die oben aufgeführten Weiterverwendungen des Materials die Einwilligung des jeweiligen Rechteinhabers einzuholen.

Weitere Details zur Lizenz entnehmen Sie bitte der Lizenzinformation auf http://creativecommons.org/ licenses/by/4.0/deed.de.

\section{Literatur}

1. Zipes DP, Camm AJ, Borggrefe $M$ et al (2006) ACC/AHA/ESC 2006 guidelines for management of patients with ventricular arrhythmias and the prevention of sudden cardiac death: a report of the American College of Cardiology/American Heart Association Task Force and the European Society of Cardiology Committee for Practice Guidelines (Writing Committee to Develop guidelines for management of patients with ventricular arrhythmias and the prevention of sudden cardiac death) developed in collaboration with the European Heart Rhythm Association and the Heart Rhythm Society. Europace 8(9):746-837. https:// doi.org/10.1093/europace/eul108 (published Online First: 2006/08/29)

2. Priori SG, Blomström-Lundqvist C, Mazzanti A et al (2015) 2015 ESC Guidelines for the management of patients with ventricular arrhythmias and the prevention of sudden cardiac death: The Task Force for the Management of Patients with Ventricular Arrhythmias and the Prevention of Sudden Cardiac Death of the European Society of Cardiology (ESC)Endorsed by: Association for European Paediatric and Congenital Cardiology (AEPC). Europace 17(11):1601-1687. https://doi org/10.1093/europace/euv319

3. Zhan C, Baine WB, Sedrakyan A et al (2008) Cardiac device implantation in the United States from 1997 through 2004: a population-based analysis. J Gen Intern Med 23(Suppl 1):13-19. https://doi.org/10. 1007/s11606-007-0392-0

4. Bogossian H, Frommeyer G, Hochadel $M$ et al (2019) Single chamber implantable cardioverter defibrillator compared to dual chamber implantable cardioverter defibrillator: less is more! Data from the German Device Registry. Clin Res Cardiol. https://doi.org/10.1007/s00392-019-01584-X

5. Owens WD, Felts JA, Spitznagel EL Jr. (1978) ASA physical status classifications: a study of consistency of ratings. Anesthesiology 49(4):239-243. https://doi.org/10.1097/00000542-19781000000003

6. Nowak B, Misselwitz B, Przibille O et al (2017) Is mortality a useful parameter for public reporting in pacemaker implantation? Results of an obligatory external quality control programme. Europace 19(4):568-572. https://doi.org/10.1093/ europace/euw079

7. Moss AJ, Zareba W, Hall WJ et al (2002) Prophylactic implantation of a defibrillator in patients with 
myocardial infarction and reduced ejection fraction. N Engl J Med 346(12):877-883. https:// doi.org/10.1056/NEJMoa013474

8. Hohnloser SH, Kuck KH, Dorian P et al (2004) Prophylactic use of an implantable cardioverterdefibrillator after acute myocardial infarction. N Engl J Med 351(24):2481-2488. https://doi.org/ 10.1056/NEJMoa041489

9. Steinbeck G, Andresen D, Seidl K et al (2009) Defibrillator implantation early after myocardial infarction. N Engl J Med 361(15):1427-1436. https://doi.org/10.1056/NEJMoa0901889

10. Connolly SJ, Hallstrom AP, Cappato R et al (2000) meta-analysis of the implantable cardioverter defibrillator secondary prevention trials. AVID, CASH and CIDS studies. Antiarrhythmics vs Implantable Defibrillator study. Cardiac arrest study hamburg . Canadian Implantable Defibrillator study. Eur Heart J 21(24):2071-2078. https://doi. org/10.1053/euhj.2000.2476 (published Online First:2000/12/05

11. van der Heijden AC, Borleffs CJ, Buiten MS et al (2015) The clinical course of patients with implantable cardioverter-defibrillators: Extended experience on clinical outcome, device replacements, and device-related complications. Heart Rhythm 12(6):1169-1176. https://doi.org/ 10.1016/j.hrthm.2015.02.035

12. Moss AJ, Schuger C, Beck CA et al (2012) Reduction in inappropriate therapyand mortality through ICD programming. N Engl J Med 367(24):2275-2283. https://doi.org/10.1056/NEJMoa1211107

13. Kloppe A, Proclemer A, Arenal A et al (2014) Efficacy of long detection interval implantable cardioverter-defibrillator settings in secondary prevention population: data from the Avoid Delivering Therapies for Nonsustained Arrhythmias in ICD Patients III (ADVANCE III) trial. Circulation 130(4):308-314. https://doi.org/10.1161/ circulationaha.114.009468

14. DewlandTA, PellegriniCN, Wang Yetal(2011)Dualchamber implantable cardioverter-defibrillator selection is associated with increased complication rates and mortality among patients enrolled in the NCDR implantable cardioverter-defibrillato registry. J Am Coll Cardiol 58(10):1007-1013. https://doi.org/10.1016/j.jacc.2011.04.039

15. Markewitz A (2019) Annual report 2017 of the German pacemaker- and defibrillator register-Part 2: implantable cardioverter defibrillators (ICD). : Working group on Cardiac pacemaker and implantable cardioverter-defibrillators at the IQTIG - Institute of Quality Assurance and Transparency in Healthcare. Herzschrittmacherther Elektrophysiol 30(4):389-403. https://doi.org/ 10.1007/s00399-019-00648-9 (published Online First:2019/11/11)

16. Klug D, Balde M, Pavin D et al (2007) Risk factors related to infections of implanted pacemakers and cardioverter-defibrillators: results of a large prospective study. Circulation 116(12):1349-1355. https://doi.org/10.1161/ circulationaha.106.678664

17. Al-Khatib SM, Greiner MA, Peterson ED et al (2009) Patient and implanting physician factors associated with mortality and complications after implantable cardioverter-defibrillator implantation, 2002-2005. Circ Arrhythm Electrophysiol 1(4):240-249. https://doi.org/10.1161/circep.108 777888

18. Ohlow MA, Lauer B, Brunelli $M$ et al (2013) Incidence and predictors of pericardial effusion after permanent heart rhythm device implantation: prospective evaluation of 968 consecutive patients. Circ J 77(4):975-981. https://doi.org/10 . 1253/circj.cj-12-0707

19. MacFadden DR, Tu JV, Chong A et al (2009) Evaluating sex differences in population-based utilization of implantable cardioverter-defibrillators: role of cardiac conditions and noncardiac comorbidities. Heart Rhythm 6(9):1289-1296. https://doi.org/10 1016/j.hrthm.2009.05.017

20. Payne T, Waller J, Kheda M et al (2020) Efficacy of implantable cardioverter-defibrillators for secondary prevention of sudden cardiac death in patients with end-stage renal disease. J Innov Card Rhythm Manag 11(8):4199-4208. https://doi.org/ 10.19102/icrm.2020.110803

21. Willems S, Tilz RR, Steven D et al (2020) Preventive or deferred ablation of ventricular tachycardia in patients with Ischemic cardiomyopathy and implantable defibrillator (BERLIN VT): a multicenter randomized trial. Circulation. https://doi.org/10. 1161/circulationaha.119.043400

22. Zhou Y, Zhao S, Chen K et al (2019) Risk of subsequent ventricular arrhythmia is higher in primary prevention patients with implantable cardioverter defibrillator than in secondary prevention patients. BMC Cardiovasc Disord 19(1):230. https://doi.org/10.1186/s12872-0191218-9

23. Køber L, Thune JJ, Nielsen JC et al (2016) Defibrillator implantation in patients with nonischemic systolic heart failure. N Engl J Med 375(13):1221-1230. https://doi.org/10.1056/ NEJMoa1608029

24. Frommeyer G, Andresen D, Ince H et al (2019) Can we rely on Danish? Real-world data on patients with nonischemic cardiomyopathy from the German Device Registry. Heart Vessels 34(7):1196-1202. https://doi.org/10.1007/s00380-018-01337-2
Hier steht eine Anzeige. Springer 